\title{
Respiratory mutations lead to different pleiotropic effects on OXPHOS complexes in yeast and in human cells
}

\author{
Sophie Marsy ${ }^{\mathrm{a}}$, Paule Frachon ${ }^{\mathrm{b}}$, Geneviève Dujardin ${ }^{\mathrm{a}}$, Anne Lombès ${ }^{\mathrm{b}}$, Claire Lemaire ${ }^{\mathrm{a}, *}$ \\ ${ }^{a}$ CNRS, Centre de Génétique Moléculaire, Avenue de la Terrasse, 91198 Gif-sur-Yvette cedex, France \\ b INSERM, U582; AP-HP, Hôpital de La Salpêtrière;UPMC-Paris6, Paris F-75013, France
}

Received 23 July 2008; revised 5 September 2008; accepted 10 September 2008

Available online 18 September 2008

Edited by Vladimir Skulachev

\begin{abstract}
Pleiotropic effects in the oxidative phosphorylation pathway (OXPHOS) were investigated in yeast respiratory mutants and in cells from patients with OXPHOS genetic alterations. The main differences between yeast and human cells were (1) the site of the primary defect that was associated with pleiotropic effects, yeast complex $\mathrm{V}$ and human complex IV, and (2) the nature of the complex targeted by the secondary effect, yeast complex IV and human complex I. The pleiotropic effects did not correlate with the organization of OXPHOS into supercomplexes and their functional consequences appeared to be a slowing down of the respiratory chain in order to avoid either an increase in the membrane potential or the accumulation of reduced intermediary components of the respiratory chain. (c) 2008 Federation of European Biochemical Societies. Published by Elsevier B.V. All rights reserved.
\end{abstract}

Keywords: Mitochondria; Oxidative phosphorylation; Respiratory complex; Saccharomyces cerevisiae;

Homo sapiens

\section{Introduction}

The mitochondrial respiratory chain (complexes I to IV) couples electron transfer to the generation of a proton gradient, which is then dissipated via complex V (ATP synthase) to produce ATP. In mammals and plants, the electron transfer chain comprises NADH quinone oxidoreductase (complex I), succinate quinone oxidoreductase (complex II), ubiquinol cytochrome $c$ oxidoreductase (complex III) and cytochrome $c$ oxidase (complex IV). In the yeast Saccharomyces cerevisiae, complex I is absent and replaced by three NADH dehydrogenases.

The association of respiratory complexes in larger order structures named supercomplexes has been demonstrated by the use of mild detergents, such as digitonin, and blue native gel electrophoresis (BN-PAGE) [1,2] for review. In mammals and plants, supercomplexes formed by complexes I and III, and by complexes I, III and IV have been described. The structure of the supercomplex $\mathrm{I}+\mathrm{III}_{2}$ of Arabidopsis at a resolution of $18 \AA$ has been obtained [3]. In yeast, supercomplexes III-IV and a dimer of ATP synthase have been visualized by electron microscopy $[4,5]$, the latter being important for the formation of cristae [6]. According to the solid state model (see [7] for re-

\footnotetext{
*Corresponding author. Fax: +33 (0) 169823160.

E-mail address: lemaire@cgm.cnrs-gif.fr (C. Lemaire).
}

view), these associations in supramolecular structures imply that alteration of one of the respiratory complexes could affect the stability of other respiratory complexes. Indeed, such pleiotropic effects have been observed with complex III and V defects in $S$. cerevisiae [8-10] and with cytochrome $b$ and COX10 defects in mammals [11,12]. To determine if the pleiotropic effects are governed by specific rules, we undertook an analysis of 12 yeast mutants and 9 human skin fibroblasts with diverse defects in the OXPHOS by combining BN-PAGE analysis, cytochrome spectrum recordings and OXPHOS activity measurements. The yeast mutants carried deletions of either a gene encoding an assembly factor (BCS1 for complex III; $S H Y 1, S C O 1$ and SCO2 for complex IV) or a structural gene of a respiratory complex subunit ( $C Y T 1, R I P 1$ and $C O R I$ for complex III; $C O X 4$ and $C O X 9$ for complex IV and $A T P 1$, $A T P 2$ and $A T P 15$ for complex V). The $\triangle a t p 1, \triangle a t p 2$ and $\triangle a t p 15$ mutants were all altered in a structural subunit of $\mathrm{F} 1$ but the $\Delta a t p 15$ mutant is the only one to exhibit a proton leakage [13]. The human fibroblasts had alterations in either a structural gene (ND5 and NDUFS4 for complex I and ATP6 for complex V) or an assembly protein (BCS1 for complex III and SURF1 and SCO2 for complex IV). The gene SURF1 is the human homolog of the yeast gene SHY1.

\section{Materials and methods}

Saccharomyces strains Arip1, Abcs1, Acyt1, Acor1, Acox4, Aatp2 and $4 a t p 15$ were in the W303 nuclear background (MAT a ade2-1 ura3-1 his3-11, 15 trp1-1 leu2-3,112 can1-100). Strains 4sco1, 4sco2, $\Delta \operatorname{shy} 1, \Delta \operatorname{cox} 9$ and $\triangle a t p 1$ were from Euroscarf and were derived from

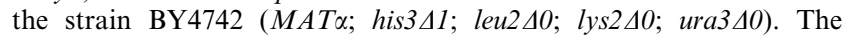
strains were grown in complete galactose medium (YPGAL) and yeast mitochondria were prepared by differential centrifugations as described in [14]. The production of petite mutants with either absence (rho ${ }^{0}$ mutants) or deletion (rho-mutants) of their mitochondrial genomes, was systematically checked by crossing the cells to a rho ${ }^{0}$ strain and testing the growth of the diploids on non-fermentable substrate. This was important as petite accumulation would lead to a non-specific pleiotropic effect on complexes III, IV and V.

Human skin fibroblasts were derived from forearm skin biopsies according to routine procedures and cultured in $4.5 \mathrm{~g} / \mathrm{L}$ glucose DMEM medium with $10 \%$ fetal calf serum, $200 \mu \mathrm{M}$ uridine and $1 \mathrm{mM}$ pyruvate. Patients' age at diagnosis, clinical presentation and genetic alteration are summarized in Table 1 .

The respiratory chain activities of cultured skin fibroblasts were analyzed by ATP measurement, polarography and spectrophotometric assays as reported in [15]. BN-PAGE was carried out according to Schagger and von Jagow [16] with slight modifications as described in [14] and was used for the separation of the respiratory complexes on $5-10 \%$ or $6-15 \%$ polyacrylamide gradient gels. Yeast mitochondria were solubilized either with $2 \%$ digitonin or $2 \%$ lauryl maltoside. 
Table 1

Age at diagnosis, clinical presentation and genetic alterations of the patients from whom the skin fibroblasts were derived

\begin{tabular}{|c|c|c|c|}
\hline Patient & Age at diagnosis & Clinical presentation & Genetic cause \\
\hline hI-1 (M) & 3 months & Leigh syndrome & NDUFS4 c. $358+1 \mathrm{G}>\mathrm{A}, \mathrm{hmz}$ \\
\hline hI-2 (F) & 3 months & Leigh syndrome & $M T-N D 5 \mathrm{~m} .13513 \mathrm{G}>\mathrm{A}$, proportion $>95 \%$ \\
\hline hIII-1 (F) & 1 month & Multi-visceral failure & $B C S 1$ c. $830 \mathrm{G}>\mathrm{A}, \mathrm{p} . \operatorname{ser} 277 \mathrm{asp}, \mathrm{hmz}$ \\
\hline hIV-1 (F) & 48 months & Leigh syndrome & SURF1 c.842T > C, p.2leu281pro and c.55 + 31_delTGCGGGG, hmz \\
\hline hIV-2 (F) & 12 months & Leigh syndrome & $S U R F 1$ c.312delTCTGCCAGCCinsAT and c.752-3C $<\mathrm{G}$ \\
\hline hIV-3 (M) & 12 months & Leigh syndrome & $S U R F 1$ c. $370 \mathrm{G}>$ A p.gly 124 arg and c. $588+1 \mathrm{G}>\mathrm{A}$ and c. $752-3 \mathrm{C}<\mathrm{G}$ \\
\hline hIV-4 (M) & 1 month & Cardiopathy, hypotonia & Sco2 c.423G > A, p.glu140lys and c.511C > T, p.arg171trp \\
\hline $\mathrm{hV}-1(\mathrm{~F})$ & 35 years & Leigh syndrome & $M T-A T P 6 \mathrm{~m} .8993 \mathrm{~T}>\mathrm{C}$, proportion $>95 \%$ \\
\hline $\mathrm{hV}-2(\mathrm{~F})$ & 10 years & Leigh syndrome & $M T$ - $A T P 6 \mathrm{~m} .8993 \mathrm{~T}>\mathrm{C}$, proportion $>95 \%$ \\
\hline
\end{tabular}

Digitonin treated fibroblasts were solubilized with 1\% lauryl maltoside [17]. Western blots of blue native gels were performed as described in [14] using polyclonal antibodies against human $\mathrm{Nd} 1 \mathrm{p}$ (complex I) and against human Cox $2 p$ (complex IV) produced in the laboratory of Dr. A. Lombès, against human Core $2 p$ (complex III) kindly provided by Dr. C. Godinot (CGMC, Lyon, France), against the yeast $\beta$ subunit (complex V) kindly provided by Dr J. Velours (IBGC, Bordeaux, France), against yeast Cytlp (complex III) produced by Dr. C. Lemaire and Dr. G. Dujardin, and against yeast Cox6p (complex IV) kindly provided by Dr. R. Poyton (University of Colorado, BO, USA). The antibodies against human $70 \mathrm{kDa}$ (complex II) were monoclonal antibodies from Molecular Probes. Immunodetection was carried out using enhanced chemiluminescence method (SuperSignal West Pico Chemiluminescent Substrate for yeast and SuperSignal West Femto Maximum Sensitivity Substrate for human fibroblasts, Pierce) and the relative amount of the different respiratory complexes was quantified using the software Multigauge provided with the LAS 3000 imaging system (Fuji).
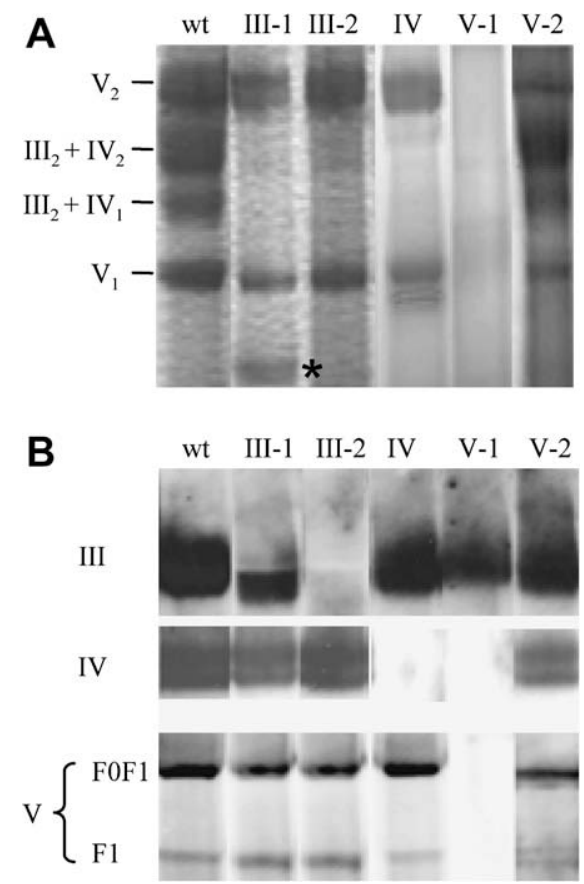

Fig. 1. Blue-Native PAGE analysis of respiratory complexes in yeast mitochondria. Mitochondria were solubilized with either digitonin (panel A) or $n$-dodecyl maltoside (panel B). Blue Native polyacrylamide gels were either stained with colloidal blue (panel A) or proteins were transferred onto nitrocellulose and immunodetected with specific polyclonal antibodies (panel B): anti- yeast Cytlp for complex III (III), anti- yeast Cox6p for complex IV (IV) and anti- $\beta$ subunit for complex $\mathrm{V}(\mathrm{V}) . \mathrm{V}_{1}$ and $\mathrm{V}_{2}$ correspond to the monomers and dimers of complex $\mathrm{V}$ respectively; $\mathrm{III}_{2}$ : dimers of complex III; $\mathrm{IV}_{1}$ and $\mathrm{IV}_{2}$ : monomers and dimers of complex IV; *: supplementary band of about $500 \mathrm{kDa}$ observed only in III-1 mutants.

\section{Results and discussion}

\subsection{Supramolecular organization and respiratory complex steady-state in yeast mutants}

Mitochondria were purified from 12 yeast mutants of complexes III, IV and V and the supramolecular organization of the three respiratory complexes was first analyzed by $\mathrm{BN}$ PAGE after digitonin solubilization to preserve the interactions between complexes (Fig. 1A). The wild-type strain (wt) showed the previously reported supramolecular organization [1] comprising four main bands corresponding to the complex $\mathrm{V}$ dimers $\left(\mathrm{V}_{2}\right)$, supercomplexes $\mathrm{III}_{2}+\mathrm{IV}_{2}$ and $\mathrm{III}_{2}+\mathrm{IV}_{1}$ and complex $\mathrm{V}$ monomers $\left(\mathrm{V}_{1}\right)$. All complex III mutants (patterns III-1 and III-2) lacked the supercomplexes $\mathrm{III}_{2}+\mathrm{IV}_{2}$ and $\mathrm{III}_{2}+\mathrm{IV}_{1}$. In addition, $\Delta$ ripl and $\Delta b c s 1$ (pattern III-1) displayed a supplementary band of about $500 \mathrm{kDa}$ (absent in $\Delta$ cyt 1 and $\Delta$ corl, pattern III-2) that was recognized by the antibodies against Cyt1p (data not shown) and might correspond to the dimeric complex III assembly intermediate lacking the FeS protein [18]. For complex IV mutants, as expected [19] the $\triangle$ sco2 strain showed a wild type pattern whereas $\Delta s h y 1, \Delta s c o l$ and $\Delta \operatorname{cox} 9$ strains showed combined defects in supercomplexes $\mathrm{III}_{2}+\mathrm{IV}_{2}$ and $\mathrm{III}_{2}+\mathrm{IV}_{1}$ (pattern IV). Finally, for complex $\mathrm{V}$ mutants, the $\Delta a t p 1$ and $\Delta a t p 2$ mutants displayed an absence of complex $\mathrm{V}$ dimers and monomers, as expected, but also of supercomplexes $\mathrm{III}_{2}+\mathrm{IV}_{2}$ and $\mathrm{III}_{2}+\mathrm{IV}_{1}$ (pattern V-1) whereas the $\Delta a t p 15$ mutant displayed a reduced amount of complex $\mathrm{V}$ dimers and monomers, and no major effect on the amount of supercomplexes $\mathrm{III}_{2}+\mathrm{IV}_{2}$ and $\mathrm{III}_{2}+\mathrm{IV}_{1}$ (pattern V-2).

In conclusion, the analysis of the supramolecular organization of respiratory complexes in these mutants showed that the deficiency of each respiratory complex induced the reduction in the supercomplexes to which it participates. Two complex $\mathrm{V}$ mutants also showed a pleiotropic effect on the steady state level of supercomplexes $\mathrm{III}_{2}+\mathrm{IV}_{2}$ and $\mathrm{III}_{2}+\mathrm{IV}_{1}$.

In order to determine if this effect was due to a defect in the supramolecular organization or to a specific lack of complex III or IV, we then checked the steady-state level of the respiratory complexes III, IV and V after solubilization of the mitochondria with laurylmaltoside, which allows separation of the different complexes. As shown in Fig. 1B, complex III or IV mutants had normal steady-state levels of the other complexes (IV and V or III and V, respectively). In addition, Aripl and $\Delta b c s 1$ complex III mutants (pattern III-1) showed a stable complex III assembly intermediate, which corresponded to the supplementary band observed in BN-PAGE (see above). In striking contrast, in addition to the absence of complex $\mathrm{V}$, the Aatpl and $\Delta a t p 2$ mutants (Fig. 1B, pattern V-1) showed 
a total lack of complex IV and a slight decrease of complex III. The absence of supercomplexes $\mathrm{III}_{2}+\mathrm{IV}_{2}$ and $\mathrm{III}_{2}+\mathrm{IV}_{1}$ observed in these mutants (Fig. 1A, pattern V-1) appeared therefore to be due to the defect of complex IV. In agreement with BN-PAGE data, the $\Delta a t p 15$ mutant (Fig. 1B, pattern V-2) showed a partial decrease in complex $\mathrm{V}$ (F0F1 and F1) but no major effect on the amount of complexes IV and III.

In conclusion, in yeast, pleiotropic effects are observed only in two complex V mutants, which exhibited combined deficiencies in complexes $\mathrm{V}$ and IV. These major effects do not seem to be related to the supramolecular organization as no interaction between complexes IV and V has been reported until now.

Alterations of complex IV have been previously described in

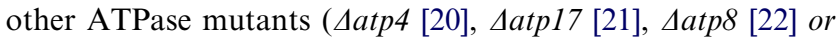
$\Delta a t p 9$ [23]). In these mutants however a high level of petite production renders the interpretation difficult. Petite production has been shown to be less than $1 \%$ in $\Delta a t p 1$ and $\Delta a t p 2$ mutants [24]. We have checked that in our conditions the petite production was also less than $1 \%$ and thus cannot explain the observed combined defect. A $\Delta a t p 6$ mutant with restricted petite accumulation has also been recently constructed [10]; it showed a significant decrease in the accumulation of complex IV related to a failure in the synthesis of Cox $1 \mathrm{p}$. The sole atp mutant ( $\triangle a t p 15)$, which does not affect complex IV, exhibits a proton leakage [13]. We therefore hypothesized that reduction of the amount of complex IV would be observed only in complex $\mathrm{V}$ mutants unable to dissipate the proton gradient. In order to test this hypothesis, we analyzed complex IV assembly in cells treated with oligomycin, a known blocker of proton translocation. As shown in Fig. 2, wild-type yeast grown in the presence of oligomycin exhibited a strong decrease of cytochromes $a$ and $a 3$ and a slight effect on the levels of cytochromes $c l$ and $b$ similar to the decreases observed in the $\Delta a t p 2$ mutant. We have checked that the petite production in our growth condition was only $10 \%$. When cells were returned to an oligomycin-free medium, the steady-state level of cyto-

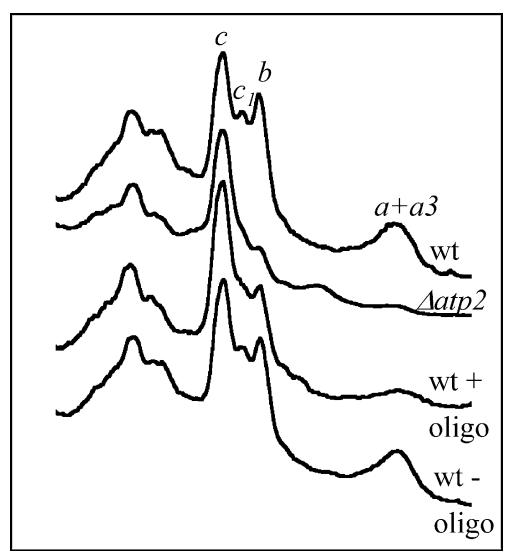

Fig. 2. Cytochrome spectra of oligomycin treated cells. Cells were grown for $15 \mathrm{~h}$ on liquid YPGAL medium. Wild-type strain (wt) and $\Delta a t p 2$ mutant were grown without oligomycin. The wild-type strain grown with $3 \mathrm{mg}$ per liter of oligomycin (wt + oligo) was rediluted in an oligomycin-free medium and grown again for $15 \mathrm{~h}(\mathrm{wt}-$ oligo). Cytochrome spectra were recorded after reduction by dithionite using a Cary 400 spectrophotometer. Cytochromes $a$ and $a 3$ are part of complex IV and cytochromes $b$ and $c 1$, of complex III. Absorption maxima of cytochromes $c, c 1, b$ and $a+a 3$ are 546, 552, 558 and $602 \mathrm{~nm}$, respectively. chromes $a$ and $a 3$ returned to normal (Fig. 2) showing that the decrease was reversible and therefore not due to secondary adaptative mutations. Blocking proton translocation thus induces a defect in complex IV and consequently a decrease of the proton gradient, which might serve to prevent the building up of a too high membrane potential.

\subsection{Steady-state of the OXPHOS complexes I, II, III, IV and V in human cells}

The low content of OXPHOS complexes in cultured skin fibroblasts makes immunoblotting a necessary approach to analyze isolated complexes solubilized by laurylmaltoside and separated by BN-PAGE. As most patients with OXPHOS genetic alterations were young children we always used the same control cells originating from a one year old girl skin biopsy. We analyzed the steady-state amount of complexes I to $\mathrm{V}$ in nine different fibroblast lines derived from patients with diverse genetic OXPHOS alterations (Table 1). Oxygen consumption, ATP production and complexes II, III, IV and V activity were also systematically measured. Complex II activity was normal in all the cells. The amount of complex II was subsequently used as a control for the amount of mitochondrial proteins. The results of immunoblotting experiments and significant alterations in activity are presented in Fig. 3. Two cell lines (hI-1 and hI-2) had alterations in structural subunits of complex I associated with a severe defect in oxygen consumption and ATP production from complex I substrates (pyruvate + malate or glutamate + malate) but normal activities using a complex II substrate (succinate + rotenone) (Fig. 3, panel complex I). BN-PAGE analysis of these cells showed alterations only in complex I: abnormal electrophoretic mobility in both cells and a $40 \%$ reduction in the amount of complex I in cells hI-2. Cells hIII-1 carried an altered BCS1 gene. They did not show a significant decrease in complex III activity in agreement with previous reports $[25,26]$. However, these cells, showed a partial but significant reduction in the steady-state level of complexes I and V (Fig. 3, panel complex III), which strengthened the relevance of the partial decrease in the rates of oxygen consumption and ATP synthesis observed with these cells, which on their own were not statistically significant.

Four cell lines had altered genes encoding assembly factors of complex IV: SURF1 (hIV-1, hIV-2 and hIV-3) or SCO2 (hIV-4). As expected, the amount of complex IV was severely reduced in these cells and correlated with the decrease in complex IV activity and oxygen consumption (Fig. 3, panel complex IV). In addition, three of the four cell lines showed a decreased steady-state amount of other respiratory complexes, which included complex I in the three cases as well as complex III in hIV-3 and complex V in hIV-4. The two cells lines with an altered complex $\mathrm{V}(\mathrm{hV}-1$ and $\mathrm{hV}-2$, Fig. 3 panel complex $\mathrm{V})$ had an identical mutation in the MT-ATP6 gene. Both cell lines had reduced amount of complex $\mathrm{V}$ and this was associated with a reduced amount of complexes I and IV in one case $(\mathrm{hV}-1)$. However the $\mathrm{hV}-1$ cell line was the only one derived from an adult patient (Table 1) and the observed reduction could have been related to difference of age between the patient and the control cells.

In conclusion, pleiotropic effects were observed in several fibroblasts lines defective for complex IV and in one line defective for $b c s 1$. They were absent in complex I defective cell lines. Definite conclusions could not be reached with complex V defects but the absence of pleiotropic effects in the most severely 
Complex I

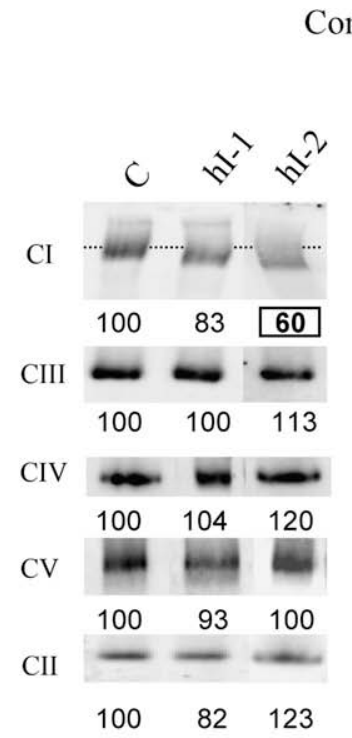

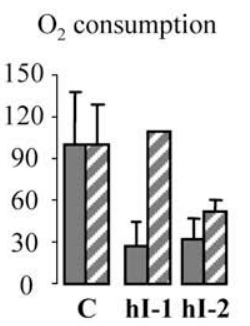

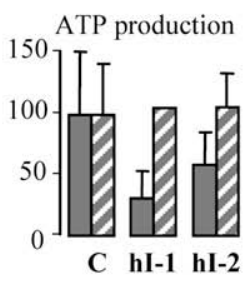

Complex IV

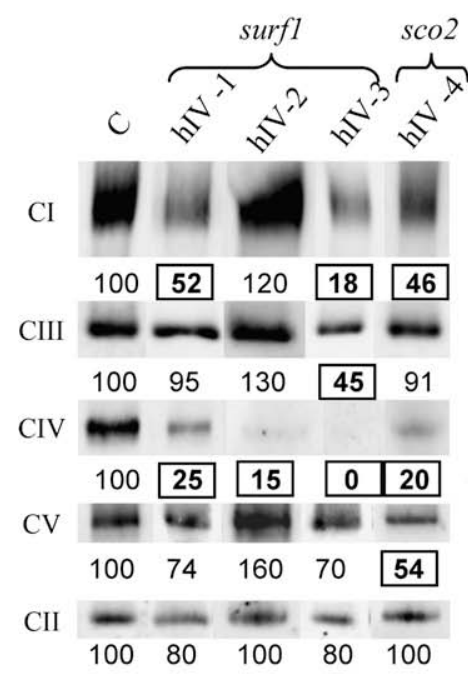

co 2
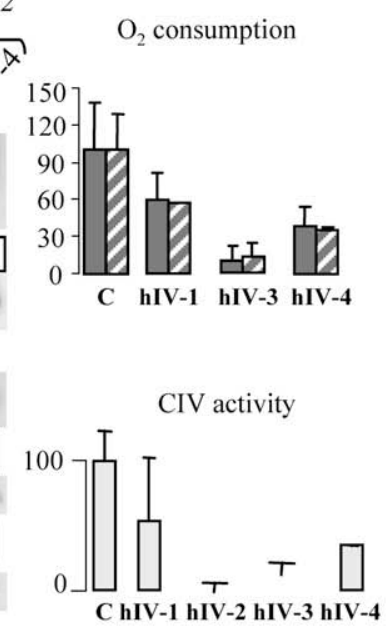

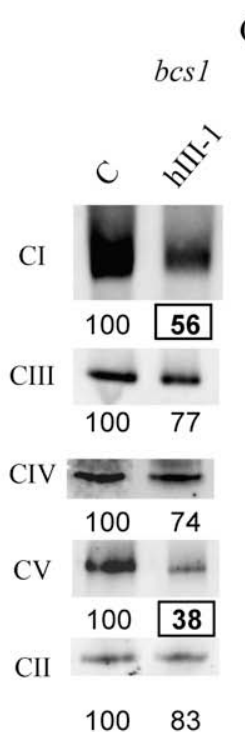

Complex III

bsl

$\mathrm{O}_{2}$ consumption
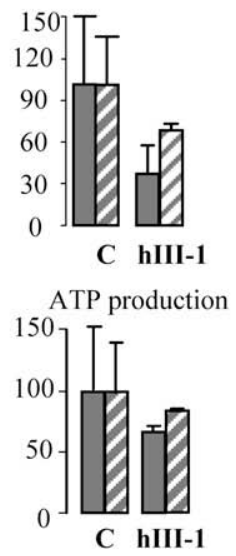

\section{Complex V}

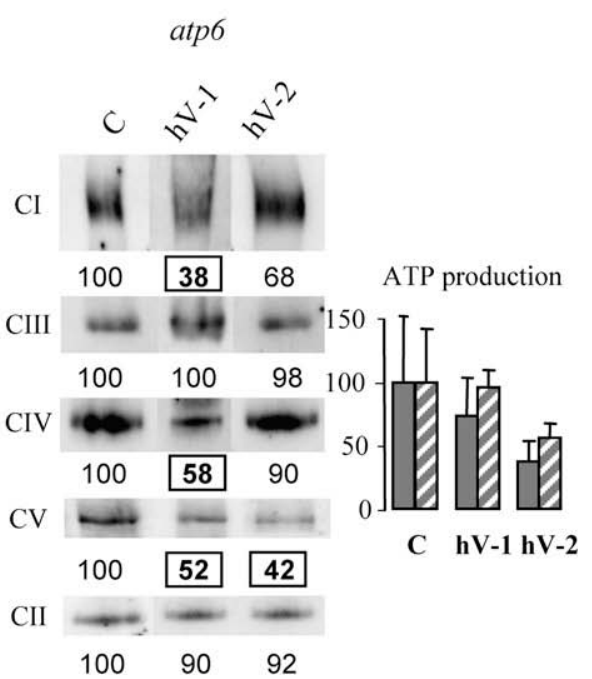

Fig. 3. Blue-Native PAGE analysis of respiratory complexes and activities in human cultured skin fibroblast mitochondria. Fibroblast mitochondria (40 $\mu \mathrm{g}$ protein) from control (C) and several patients were separated by BN-PAGE 5-10\% polyacrylamide gradients for the analysis of complexes I and $\mathrm{V}$, or on $6-15 \%$ polyacrylamide gradients for the analysis of complexes II, III and IV. Proteins were transferred onto nitrocellulose and immunodetected with anti-Nd1p subunit for complex I (CI), anti-70 kDa subunit for complex II (CII), anti-Core2p for complex III (CIII), antiCox $2 p$ for complex IV (CIV) and anti- $\beta$ subunit for complex V (CV). The dashed line indicates the electrophoretic mobility of complex I in the control. Quantification of each complex is expressed as a percentage of the control below the line. Significant modifications are boxed and noted in bold. Oxygen consumption, ATP production and CIV activity are expressed as a percentage of the control. Oxygen consumption and ATP production were measured using either complex I substrates (pyruvate + malate or glutamate + malate, results shown as grey bars) or the complex II substrate (succinate + rotenone, results shown as hatched bars).

affected cells $(\mathrm{hV}-2)$ is a striking observation. These data are important for diagnosis as they show that a concomitant alteration of complexes I and IV could be an indicator of a primary defect on complex IV assembly. The mechanisms underlying these pleiotropic effects remain to be clarified. As in yeast, they do not seem to be related to supercomplexes, as we have not observed any concomitant decrease in the complexes I, III and IV that are associated together. Again, as in yeast, these observations are compatible with the hypothesis that human cells employ mechanisms to slow down electron transfer and/ or to limit the membrane potential in the presence of certain OXPHOS defect. Targeting complex I in human cells might also have a major effect on the production of reactive oxygen species by decreasing the steady-state of reduced intermediary components of the respiratory chain. This difference could suggest that the physiological phenomenon underlying the regulation may be different between yeast and humans especially as $S$. cerevisiae does not have complex I.

In conclusion, the pleiotropic effects of an isolated OXPHOS alteration may be observed in man as in $S$. cerevisiae and they do not seem to correlate with the organization of OXPHOS complexes into supercomplexes. The functional consequences of the pleiotropic effects appear to be the slowing down of the respiratory chain in order to prevent either a too high membrane potential or the accumulation of reduced intermediary components of the respiratory chain. 
Acknowledgements: We thank J.P.di Rago and J.Velours for the gift of the strains $\triangle a t p 2$ and $\Delta a t p 15$, respectively. We are grateful to C.J. Herbert and $\mathrm{N}$. Clarke for critical reading of the manuscript, and to $\mathrm{H}$. Antonicka and C. Nouet for helpful discussions. This work was supported by a research grant from Association Française contre les Myopathies and by the Agence Nationale pour la Recherche BLAN060234 .

\section{References}

[1] Schagger, H. and Pfeiffer, K. (2000) Supercomplexes in the respiratory chains of yeast and mammalian mitochondria. EMBO J. 19 (8), 1777-1783.

[2] Boekema, E.J. and Braun, H.P. (2007) Supramolecular structure of the mitochondrial oxidative phosphorylation system. J. Biol. Chem. 282 (1), 1-4.

[3] Dudkina, N.V., Eubel, H., Keegstra, W., Boekema, E.J. and Braun, H.P. (2005) Structure of a mitochondrial supercomplex formed by respiratory-chain complexes I and III. Proc. Natl. Acad. Sci. USA 102 (9), 3225-3229.

[4] Heinemeyer, J., Braun, H.P., Boekema, E.J. and Kouril, R. (2007) A structural model of the cytochrome $c$ reductase/oxidase supercomplex from yeast mitochondria. J. Biol. Chem. 282 (16), 12240-12248.

[5] Dudkina, N.V., Sunderhaus, S., Braun, H.-P. and Boekema, E. (2006) Characterization of dimeric ATP synthase and cristae membrane ultrastructure from Saccharomyces and Polytomella mitochondria. FEBS Lett. 580 (14), 3427-3432.

[6] Paumard, P., Vaillier, J., Coulary, B., Schaeffer, J., Soubannier, V., Mueller, D.M., Brethes, D., di Rago, J.P. and Velours, J. (2002) The ATP synthase is involved in generating mitochondrial cristae morphology. EMBO J. 21 (3), 221-230.

[7] Rich, P.R. (1984) Electron and proton transfers through quinones and cytochrome $b c$ complexes. Biochim. Biophys. Acta 768 (1), 53-79.

[8] Bruel, C., Brasseur, R. and Trumpower, B.L. (1996) Subunit 8 of the Saccharomyces cerevisiae cytochrome $b c 1$ complex interacts with succinate-ubiquinone reductase complex. J. Bioenerg. Biomembr. 28 (1), 59-68.

[9] Tzagoloff, A., Akai, A. and Needleman, R.B. (1975) Assembly of the mitochondrial membrane system. Characterization of nuclear mutants of Saccharomyces cerevisiae with defects in mitochondrial ATPase and respiratory enzymes. J. Biol. Chem. 250 (20), 8228-8235.

[10] Rak, M., Tetaud, E., Godard, F., Sagot, I., Salin, B., DuvezinCaubet, S., Slonimski, P.P., Rytka, J. and di Rago, J.P. (2007) Yeast cells lacking the mitochondrial gene encoding the ATP synthase subunit 6 exhibit a selective loss of complex IV and unusual mitochondrial morphology. J. Biol. Chem. 282 (15), 10853-10864.

[11] Acin-Perez, R., Bayona-Bafaluy, M.P., Fernandez-Silva, P., Moreno-Loshuertos, R., Perez-Martos, A., Bruno, C., Moraes, C.T. and Enriquez, J.A. (2004) Respiratory complex III is required to maintain complex $\mathrm{I}$ in mammalian mitochondria. Mol. Cell 13 (6), 805-815.

[12] Diaz, F., Fukui, H., Garcia, S. and Moraes, C.T. (2006) Cytochrome $c$ oxidase is required for the assembly/stability of respiratory complex I in mouse fibroblasts. Mol. Cell. Biol. 26 (13), 4872-4881.
[13] Guélin, E., Chevallier, J., Rigoulet, M., Guérin, B. and Velours, J. (1993) ATP synthase of yeast mitochondria. Isolation and disruption of the ATP epsilon gene. J. Biol. Chem. 268 (1), 161-167.

[14] Lemaire, C. and Dujardin, G. (2008) Preparation of respiratory chain complexes from Saccharomyces cerevisiae wild-type and mutant mitochondria: activity measurement and subunit composition analysis. Meth. Mol. Biol. 432, 65-81.

[15] Guillery, O., Malka, F., Frachon, P., Milea, D., Rojo, M. and Lombes, A. (2008) Modulation of mitochondrial morphology by bioenergetics defects in primary human fibroblasts. Neuromuscul. Disord. 18, 319-330.

[16] Schagger, H. and von Jagow, G. (1991) Blue native electrophoresis for isolation of membrane protein complexes in enzymatically active form. Anal. Biochem. 199 (2), 223-231.

[17] Antonicka, H., Ogilvie, I., Taivassalo, T., Anitori, R.P., Haller, R.G., Vissing, J., Kennaway, N.G. and Shoubridge, E.A. (2003) Identification and characterization of a common set of complex I assembly intermediates in mitochondria from patients with complex I deficiency. J. Biol. Chem. 278 (44), 43081-43088.

[18] Cruciat, C.M., Brunner, S., Baumann, F., Neupert, W. and Stuart, R.A. (2000) The cytochrome $b c l$ and cytochrome $c$ oxidase complexes associate to form a single supracomplex in yeast mitochondria. J. Biol. Chem. 275 (24), 18093-18098.

[19] Lode, A., Paret, C. and Rodel, G. (2002) Molecular characterization of $S$. cerevisiae Sco2p reveals a high degree of redundancy with Scolp. Yeast 19 (11), 909-922.

[20] Paul, M.F., Velours, J., Arselin de Chateaubodeau, G., Aigle, M. and Guerin, B. (1989) The role of subunit 4, a nuclear-encoded protein of the F0 sector of yeast mitochondrial ATP synthase, in the assembly of the whole complex. Eur. J. Biochem. 185 (1), 163171.

[21] Spannagel, C., Vaillier, J., Arselin, G., Graves, P.V. and Velours, J. (1997) The subunit $f$ of mitochondrial yeast ATP synthasecharacterization of the protein and disruption of the structural gene ATP17. Eur. J. Biochem. 247 (3), 1111-1117.

[22] Marzuki, S., Watkins, L.C. and Choo, W.M. (1989) Mitochondrial H+-ATPase in mutants of Saccharomyces cerevisiae with defective subunit 8 of the enzyme complex. Biochim. Biophys. Acta 975 (2), 222-230.

[23] Hadikusumo, R.G., Meltzer, S., Choo, W.M., Jean-Francois, M.J., Linnane, A.W. and Marzuki, S. (1988) The definition of mitochondrial H+ ATPase assembly defects in mit- mutants of Saccharomyces cerevisiae with a monoclonal antibody to the enzyme complex as an assembly probe. Biochim. Biophys. Acta $933(1), 212-222$.

[24] Lai-Zhang, J., Xiao, Y. and Mueller, D.M. (1999) Epistatic interactions of deletion mutants in the genes encoding the F1ATPase in yeast Saccharomyces cerevisiae. EMBO J. 18 (1), 5864

[25] de Lonlay, P., Valnot, I., Barrientos, A., Gorbatyuk, M., Tzagoloff, A., Taanman, J.W., Benayoun, E., Chretien, D., Kadhom, N., Lombes, A., de Baulny, H.O., Niaudet, P., Munnich, A., Rustin, P. and Rotig, A. (2001) A mutant mitochondrial respiratory chain assembly protein causes complex III deficiency in patients with tubulopathy, encephalopathy and liver failure. Nat. Genet. 29 (1), 57-60.

[26] Fernandez-Vizarra, E., Bugiani, M., Goffrini, P., Carrara, F., Farina, L., Procopio, E., Donati, A., Uziel, G., Ferrero, I. and Zeviani, M. (2007) Impaired complex III assembly associated with BCS1L gene mutations in isolated mitochondrial encephalopathy. Hum. Mol. Genet. 16 (10), 1241-1252. 